\title{
EDITORIAL
}

\section{The dilemma of apoptosis in myelodysplasia and leukemia: a new promise of therapeutic intervention?}

\author{
MV Blagosklonny \\ Medicine Branch, National Cancer Institute, NIH, Bethesda, MD, USA
}

In this issue of Leukemia, Boudard et al' describe that the caspase inhibitor DEVD-cho restored colony formation of CFU-GM, CFU-E, and BFU-E progenitors in myelodysplastic syndrome (MDS). This can be envisioned as a basis for a novel therapeutic approach for MDS. The prospect of a new therapeutic approach for a difficult-to-cure disease is always an exciting milestone, even though many promises made are eventually proven fruitless.

Normal hematopoiesis is regulated by the coordination between proliferation, differentiation, and apoptosis. Apoptosis is cell death-mediated by caspase activation. Caspases (cysteine aspartate-specific proteases) are a family of proteases with a stringent requirement for cleavage after aspartate residues. ${ }^{2}$ Caspase activation results in rapid cell death that is usually accompanied by nuclear and DNA fragmentation, membrane blebbing and cell shrinkage, all known as morphological features of apoptosis. Apoptosis is initiated by the activation of the proximal caspases, either caspase-8, caspase- 9 or caspase-12, leading to activation of the executioner caspases such as caspase- $3,-6$ and -7 . In addition, noncaspases, including cathepsins, calpains, granzymes also have roles in promoting cell death. ${ }^{3}$

Apoptosis is often evaded by cancer and leukemia cells. ${ }^{4}$ For example, in Ph1 (+) leukemia cells, the expression of the $\mathrm{BCR}-\mathrm{ABL}$ chimeric gene prevents apoptosis in response to a variety of anticancer agents. ${ }^{5}$ Spontaneous apoptosis in leukemia cells correlates with chemotherapy-induced apoptosis and is associated with better prognosis. Chemotherapeutic drugs also induce death of normal cells causing cytopenias.

Like therapy-induced cytopenia and unlike leukemia, MDS is related to excessive apoptosis. MDS represents a spectrum of disorders and is characterized by peripheral blood cytopenias, and a tendency to evolve into acute myeloid leukemias. ${ }^{6}$ Cytopenia with a hypercellular marrow can be attributed to increased proliferation with apoptosis (ineffective hematopoiesis). Evidence indicates that apoptosis is involved in the development of MDS. ${ }^{7}$ Caspase activity is especially enhanced in 'early' MDS. ${ }^{1,8}$

MDS is divided into 'early stage' myelodysplasia (refractory anemia (RA), RA with ringed sideroblasts, RAEB with less than $10 \%$ blasts) and 'late stage' myelodysplasia (RAEB with more than $10 \%$ blasts, RAEB in transformation (RAEB-t), and acute myeloid leukemia secondary to myelodysplasia).

Boudard et al demonstrated that, caspase-3 activation is associated with 'early' MDS. Caspase- 3 activation declined in 'late' MDS and was absent in leukemia. MDS is characterized by abnormal growth of hematopoietic progenitor cells (CFUGM, CFU-E, BFU-E) in clonogenic assays, as a result of excess-

Correspondence: MV Blagosklonny, Medicine Branch, NIH, Bldg 10, Room 12 N 226, Bethesda, MD 20892, USA; Fax: 3014020172 Received 1 September 2000; accepted 6 September 2000 ive cell death. The inhibitor of caspases, DEVD-cho, increased the number of all types of colonies, especially in 'early' MDS. In some cases, the number of BFU-E and CFU-E was increased almost 50 times, thus reaching the normal values.

Recently, it has been reported that despite the well-documented activation of caspase- 3 in cells derived from patients with MDS, the caspase inhibitor Z-VAD-fmk did not increase colony formation. ${ }^{8}$ The article by Boudard et al in this issue of Leukemia suggests that DEVD-cho (but not the inhibitor used in the other study) increases colony formation. Many questions remain. What are the stimuli that cause caspase activation in MDS? The Fas/Fas ligand system is implicated in MDS. Since Fas-mediated apoptosis is initiated by activation of caspase-8, can inhibitors of caspase-8 such as IETD-cho restore colony formation?

The demonstration that sole inhibition of caspases can restore colony formation in vitro is important. In this light, it is very tempting to consider clinical applications of caspase inhibitors in the treatment of MDS. Will caspase inhibitors work in vivo? Caspase inhibitors are small peptides that mimic substrates of caspases. In animal models, caspase inhibitors abrogate apoptosis. Thus, Ac-VAD, Z-DEVD-fmk, and B-Dfmk inhibitors abrogated apoptosis in ischemic liver and fully protected rats from death. ${ }^{9}$ Z-VAD-fmk prevented Fas-induced damage of mouse liver. ${ }^{10}$ Inhibitors of caspases are currently being considered for treatment of several conditions, including stroke, meningitis and sepsis.

Some of the current therapeutic strategies for MDS and leukemia, including chemotherapy and transplantation, seem somehow similar. ${ }^{11}$ Inhibitors of caspases represent an opposing strategy. It should be noted that amifostine (a phosphorylated aminothiol), a free radical scavenger and inhibitor of TNF elaboration, has been used in the treatment of MDS, as an anti-apoptotic agent. ${ }^{6}$ However, its effectiveness is disputed. Although it is called an anti-apoptotic agent, amifostine has an indirect mechanism of action. In contrast, according to the biochemical definition of apoptosis, caspase inhibitors are straightforward inhibitors of apoptosis. Inhibition of caspases may be effective regardless of the biological nature of these diseases and in theory, may be useful in severe anaplastic anemia.

However, potential long-term outcomes resulting from treatment of MDS with caspase inhibitors are unclear. According to the current view, apoptotic cell death is increased in early MDS, declines in late MDS and is inhibited in leukemia. As noted 25 years ago, it is dangerous to restore colonies which may contain leukemic cells. ${ }^{12}$ Will treatment with caspase inhibitors result in the remission of MDS or in the development of leukemia? The dilemma between an excessive apoptosis in MDS and an inhibited apoptosis in leukemia should be kept firmly in mind.

One possibility is that apoptosis in MDS eliminates a potentially leukemic clone, and the inhibition of apoptosis pro- 
motes the development of leukemia. In this scenario, chemotherapy rather than caspase inhibitors may be indicated as the appropriate therapy. However, in treatment of bone marrow insufficiency that is caused by acute toxicities there is little reason to expect the development of leukemia. Also, caspase inhibitors could be advocated for severe aplastic anemia because the risk of progression to acute leukemia is lower in aplastic anemia.

Another possibility is that excessive apoptosis in MDS prompted the selection of a leukemic clone. ${ }^{13}$ Clonal outgrowths in MDS and secondary leukemia may be the result of strong selective pressure on stem and/or progenitor cells. In this scenario, inhibitors of caspases will not only restore hematopoiesis, but also will delay or even prevent development of leukemia.

Given that hemopoietic stem-cell transplantation is currently the only potentially curative therapy, ${ }^{14}$ consideration of caspase inhibitors for the treatment of MDS is very tempting.

\section{References}

1 Boudard, Sordet O, Vasselon C, Revol V, Berthéas M-F, Freyssenet D, Viallet A, Piselli S, Guyotat D, Campos L. Expression and activity of caspases 1 and 3 in myelodysplastic syndromes. Leukemia 2000; 14: 2045-2051.

2 Fadeel B, Orrenius S, Zhivotovsky B. The most unkindest cut of all: on the multiple roles of mammalian caspases. Leukemia 2000; 14: 1514-1525.

3 Johnson DE. Noncaspase proteases in apoptosis. Leukemia 2000; 14: 1695-1703.

4 Reed JC. Dysregulation of apoptosis in cancer. J Clin Oncol 1999; 17: 2941-2954.
5 Samali A, Gorman AM, Cotter TG. Role of Bcr-Abl kinase in resistance to apoptosis. Adv Pharmacol 1997; 41: 533-552.

6 Rosenfeld C, List A. A hypothesis for the pathogenesis of myelodysplastic syndromes: implications for new therapies. Leukemia 2000; 14: 2-8.

7 Mundle SD, Venugopal P, Cartlidge JD, Pandav DV, Broady-Robinson L, Gezer S, Robin EL, Rifkin SR, Klein M, Alston DE, Hernan$\operatorname{dez}$ BM, Rosi D, Alvi S, Shetty VT, Gregory SA, Raza A. Indication of an involvement of interleukin-1 beta converting enzyme-like protease in intramedullary apoptotic cell death in the bone marrow of patients with myelodysplastic syndromes. Blood 1996; 88: 2640-2647.

8 Bouscary D, Chen Y, Guesnu M, Picard F, Viguier F, Lacombe C, Dreyfus F, Fontenay Roupie M. Activity of the caspase-3/CPP32 enzyme is increased in 'early stage' myelodysplastic syndromes with excessive apoptosis, but caspase inhibition does not enhance colony formation in vitro. Exp Hematol 2000; 28: 784-791.

9 Cursio R, Gugenheim J, Ricci JE, Crenesse D, Rostagno P, Maulon L, Saint-Paul MC, Ferrua B, Auberger AP. A caspase inhibitor fully protects rats against lethal normothermic liver ischemia by inhibition of liver apoptosis. FASEB J 1999; 13: 253-261.

10 Chandler JM, Cohen GM, MacFarlane M. Different subcellular distribution of caspase-3 and caspase-7 following Fas-induced apoptosis in mouse liver. J Biol Chem 1998; 273: 10815-10818.

11 Hellstrom-Lindberg E. Treatment of adult myelodysplastic syndromes. Int I Hematol 1999; 70: 141-154.

12 Killmann SA. Preleukemia: does it exist? Nouv Rev Fr Hematol Blood Cells 1976; 17: 81-105.

13 Lensch MW, Rathbun RK, Olson SB, Jones GR, Bagby GCJ. Selective pressure as an essential force in molecular evolution of myeloid leukemic clones: a view from the window of Fanconi anemia. Leukemia 1999; 13: 1784-1789.

14 Deeg HJ, Appelbaum FR. Hemopoietic stem cell transplantation for myelodysplastic syndrome. Curr Opin Oncol 2000; 12: 116120. 